\title{
Heterogeneous ice nucleation on dust particles sourced from 9 deserts worldwide - Part 1: Immersion freezing
}

Yvonne Boose $^{1}$, André Welti ${ }^{1,2}$, James Atkinson ${ }^{1}$, Fabiola Ramelli ${ }^{1}$, Anja Danielczok ${ }^{3}$, Heinz G. Bingemer ${ }^{3}$, Michael Plötze ${ }^{4}$, Berko Sierau ${ }^{1}$, Zamin A. Kanji ${ }^{1}$, and Ulrike Lohmann ${ }^{1}$

${ }^{1}$ Institute for Atmospheric and Climate Science, ETH Zürich, Zürich, Switzerland

${ }^{2}$ now at Leibniz Institute for Tropospheric Research, Leipzig, Germany

${ }^{3}$ Institute for Atmospheric and Environmental Sciences, J. W. Goethe-University, Frankfurt am Main, Germany

${ }^{4}$ Institute for Geotechnical Engineering, ETH Zürich, Zürich, Switzerland

Correspondence to: Y.Boose (yvonne.boose@env.ethz.ch) and Z. A. Kanji (zamin.kanji@env.ethz.ch) 
Table 1. GPS coordinates of sample collection sites.

\begin{tabular}{cccc}
\hline Collection site & type & latitude & longitude \\
\hline Atacama & sieved & -22.03 & -67.88 \\
Atacama & milled & -22.03 & -67.88 \\
Australia & milled & -25.32 & 131.63 \\
Crete & airborne & 35.34 & 25.67 \\
Dubai & sieved & 24.83 & 55.66 \\
Egypt & airborne & 28.93 & 33.21 \\
Great Basin & sieved & 37.16 & -116.49 \\
Peloponnese & airborne & 37.97 & 23.78 \\
Israel & sieved & 30.84 & 34.79 \\
Israel & milled & 30.84 & 34.79 \\
Mojave & sieved & 36.71 & -117.17 \\
Morocco & milled & 31.21 & -3.99 \\
Namib & sieved & 18.01 & 16.01 \\
Tenerife & airborne & 28.31 & -16.50 \\
\hline
\end{tabular}

